\title{
Evaluating the Impact of Alcohol Abstinence on the Cognitive Functioning of Adults Diagnosed with Alcohol Use Disorder
}

\author{
Erengai E. Mofokeng \& Sonja N. Mostert \\ University of Pretoria, Pretoria, SOUTH AFRICA \\ Faculty of Humanities
}

Received: 29 September 2021 - Accepted: 9 November 2021 - Published Online: 9 December 2021

\begin{abstract}
Research supports the relation between chronic alcohol use and progressive cognitive impairment but alcohol rehabilitation facilities mainly focus on psycho-social factors with limited emphasis on cognitive impairment and recovery. This study evaluated the impact of alcohol abstinence on cognitive functioning; specifically, visuospatial attention, working memory and abstract reasoning on two different occasions using the WAIS-IVSA battery. A pre-test-post-test design was used to assess patients diagnosed with Alcohol Use Disorder (AUD). Differences in cognitive functioning from phase 1 (3-4 days after admission) and phase 2 (14 days after phase 1) were measured. A paired samples t-test was conducted using SPSS version 27. A statistically significant increase in AUD patients' visuospatial scores was found when comparing phase 1 and $2(\mathrm{M}=7.11, \mathrm{SD}=2.07), \mathrm{t}(8)=3.42, \mathrm{p}=.009$. No statistically significant differences were observed for working memory and abstract reasoning. We conclude that the findings can be used to guide, and improve the development of future intervention programs to emphasize the value of cognitive recovery.
\end{abstract}

Keywords: abstract reasoning, alcohol abstinence, alcohol use disorder, visuospatial attention, working memory.

\section{Introduction}

The World Health Organization recognises alcoholism as a chronic progressive disease (WHO, 2021) and Alcohol use disorder (AUD) is characterised by an intense desire to consume alcohol and, where attempts to limit or control the use of alcohol, has been unsuccessful. Le Berre et al. (2017) explain that alcoholism is a "...complex, multidimensional, multidetermined disorder" (p.1432). As a result, the structural and functional influence of alcohol abuse on the brain can be mediated by several factors (Velayudhan \& Saraswathy, 2020). Neuropsychological profiles related to alcoholism vary due to the diverse origin of alcoholism and its outcomes which are influenced by family history, mental and physical health, gender and age (Oscar-Berman et al., 2014; Velayudhan \& Saraswathy, 2020). The period of alcohol use, and frequency of intake, can also impact different brain regions and functioning, differently. Similarly, alternating periods of withdrawal, abstinence, and relapse also contribute complications (Le Berre et al., 2017).

(C) Authors. Terms and conditions of Creative Commons Attribution 4.0 International (CC BY 4.0) apply. Correspondence: Sonja N. Mostert, University of Pretoria, Faculty of Humanities, Pretoria, SOUTH AFRICA. E-mail: sonja.mostert@up.ac.za. 
E. E. Mofokeng \& S. N. Mostert - Evaluating the Impact of Alcohol Abstinence...

- The same group of patients was assessed on two different occasions: A baseline assessment and a post-test assessment. The degree of impairment and recovery was thus observed amongst the same AUD sample.

- The findings have practical and theoretical value. It contributes to the knowledge base regarding the impact of alcohol abuse on cognitive functioning:

- The study emphasises the significance of measuring cognitive functioning and the importance of determining AUD patients' level of cognition.

- Practically the study offers valuable information that may be used to guide future interventions specifically aimed at improving alcohol-related cognitive impairment.

- Cognitive evaluation is important for both the assessment and treatment of AUD patients.

- AUD patients need cognitive support post-rehabilitation to minimize relapse.

Alcohol abuse results in several health-compromising consequences, leading to early mortality and numerous chronic health conditions (Brennan et al., 2020; Devere, 2016). It is linked to various neurophysiological and cognitive alterations, including; the ability to learn, memory difficulties, diminished decision-making and challenges with motor skills (Ahmadi et al., 2013; Fein et al., 2006; Ioime et al., 2018; Kopera et al., 2012; Velayudhan \& Saraswathy, 2020). In conjunction, patients may also present with psychological symptoms like anxiety and depression (Erdozain et al., 2014; Woods et al., 2016). Despite awareness of the negative physical, psychological, occupational, or social consequences experienced, patients continue to abuse alcohol. To enable recovering alcoholics to maintain abstinence effective cognitive functioning is needed (Le Berre et al., 2017).

Several studies suggest that chronic alcohol use is linked to progressive cognitive impairment (Banerjee, 2014; Bates et al., 2013; Bernadin et al., 2014; Erdozain et al., 2014; Mocaiber et al., 2011). The severity of cognitive impairment, however, varies and several factors impact the extent of damage (Batman, 2015; Le Berre et al., 2017). Impairment stemming from AUD is especially prominent in higher-order cognitive functions, such as abstract reasoning, visuospatial processing and problem-solving (Bagga et al., 2014; Kopera et al., 2012). AUD is not only characterised by a lack of self-control but also relates to deficits in cognitive control, which is needed for goal-directed behaviour (Breukelaar et al., 2017; Molnar et al., 2018). Ioime et al. (2018) emphasise the value of the "cognitive emotional-affective" domain and the adverse impact of alcohol use on this neural mechanism (p. 505). Cognitive impairment can impact the extent to which AUD patients manage their addiction and related emotions, and increase the possibility of relapse. Adequate cognitive functioning and control are thus considered a prerequisite for effective psychological treatment (Ioime et al., 2018). The specific cognitive functions vulnerable to damage due to alcohol abuse and how abstinence may contribute to cognitive recovery, is, however, still unclear (Stavro et al., 2013).

Research supports the notion that cognitive recovery is possible after a period of abstinence (Fein et al., 2006) with several cognitive domains returning to normal. There is, however, evidence of the contrary in that certain cognitive impairments persist even after long periods of abstinence (Ioime et al., 2018; Velayudhan \& Saraswathy, 2020). A review by OscarBerman et al. (2014) found that abstaining from alcohol, after a period of abuse, shows that the brain appears to re-organise itself to offer compensation for structural and behavioural impairment. Similar findings were reported by Fein et al. (2006) and Zehra et al. (2019).) Given that disagreement is still evident, more research can be valuable to understand the neurocognitive recovery associated with abstinence (Brennan et al.,2020; Fein et al., 2006). The validity of findings is often questioned based on several factors, including the measures used, the timing of assessment, the sample included and the period of abstinence. The degree to which cognitive functions recover is thus not yet well conceptualised. 
Ioime et al. (2018) highlighted that research supports the detrimental effects of alcohol abuse on cognition, but the nature of the impact on specific brain regions or the global impact is still deliberated. Following this, the influence of continued abstinence and the nature of cognitive recovery also remains unclear. Assessing the cognitive functioning of AUD patients is imperative, not only to determine the level of impairment but also to ascertain those patients have the ability to benefit from psychosocial treatment. The importance of assessing cognition, and including interventions to improve cognitive functioning is needed as "...cognitive impairment reduces the effectiveness of psychological treatments" (Ioime et al., 2018: 505). Steps thus need to be included to improve cognitive functioning and the value of abstinence in strengthening these functions need to be explored. Improving our understanding of AUD cognitive related decline is valuable for both public health and the understanding of addiction (Ahmadi et al., 2013).

Evidence of improvement in dysfunction, caused by alcohol abuse, during recovery suggests that the brain is capable of restructuring (Velayudhan \& Saraswathy, 2020). Recovery of diverse cognitive functions is dependent on the nature of the dysfunction as some functions improve after a short period of abstinence but others may require longer periods of recovery. Certain cognitive functions may also persist despite long periods of sobriety (Fein et al., 2006; Ioime et al., 2018). A focus on the change of specific cognitive functions on the same sample of AUD patients from baseline is needed to obtain a more comprehensive understanding of the extent of cognitive damage as well as the level of improvement after recovery.

The primary neuropsychological domains assessed in people with AUD have been memory, executive functions emotion, psychosocial skills, visuospatial cognition, and psychomotor abilities (Oscar-Berman et al., 2014). Given the diverse implications of alcohol use on cognitive functioning and the lack of consensus regarding the nature of recovery, the current study focused on the impact of abstinence, as part of a rehabilitation programme, on three prominent cognitive functions, namely; visuospatial attention (VA); abstract reasoning (AR), and working memory (WM).

VA involves the selection of information in relation to where stimuli are in space (Zirnsak \& Moore, 2015). It is an adaptive mechanism that enables people to follow set goals while reacting to and processing stimuli in the surrounding environment (Doruk et al., 2018). VA has important practical implications in relation to driving ability, for example (Buddy, 2020). Evidence shows that chronic alcohol use does not impair all brain regions involved in selective attention equally (Buddy, 2020; do Canto-Pereira et al., 2007; Kopera et al., 2012). Fein et al. (2006) found that spatial processing is one of the main cognitive functions weakened by alcohol abuse. Even after periods of abstaining from alcohol, dysfunction related to visuospatial abilities persists (Buddy, 2020).

Zehra et al. (2019) emphasised the limited understanding of the impact of alcohol use on attention. In their study, 19 newly abstinent patients diagnosed with AUD and 23 healthy controls were assessed on a VA task using fMRI. No behavioural differences were found between the groups, but the results indicated decreased activation in VA brain regions in the AUD patients compared to controls. Similarly, a study by do Canto-Pereira et al. (2007) found that acute alcohol intoxication impairs the ability to disengage attention from a gaze point (do Canto-Pereira et al., 2007). Likewise, Ioime et al. (2018) found that the VA abilities in alcoholic dependent participants demonstrate a slower recovery rate as their sample continued to display problems with VA tasks despite several weeks of recovery. Further exploration about the impact of alcohol use and abstinence on VA is reasonable to elucidate the nature of this cognitive function in relation to recovery.

AR is another prominent cognitive domain impaired by alcoholism and refers to the ability to readily identify patterns, logical rules and trends in stimuli followed by integration and application to solve problems (Astorga, 2013). Bagga et al. (2014) used fMRI scans to study the 
neuropsychological basis of poor AR abilities in alcohol-dependent participants. The results showed that alcohol-dependent participants used additional brain areas to execute behavioural tasks in comparison to controls. There was no significant difference in the response accuracy between the groups, but the findings suggest that for alcohol-dependent participants, more effort and activation of additional brain areas are needed to complete tasks. Buddy (2020) echoed these findings emphasising that alcoholics rely on more complex higher-order functions to aid them in task performance.

In conjunction with $\mathrm{AR}$, and VA, WM also plays a major role in goal-directed behaviour. This memory system enables the retention and manipulation of information (Chai et al., 2018; Cowan, 2014; Deshpande, 2015), necessary for successful task completion. Various studies have indicated that WM is one of the key components impaired by alcohol (Deshpande, 2015; Velayudhan \& Saraswathy, 2020). Results from a study by Saults et al. (2007), indicated that WM abilities, specifically auditory and visual arrangements, are impaired by prolonged alcohol abuse. Kopera et al. (2012) found similar results and maintained that WM is impaired in short-term abstinent alcoholics. Lechner et al. (2015) assessed the relationship between drinking behaviour and the consequent changes in WM. It was reported that a person who starts drinking with the intention to stop or to avoid risks associated with uncontrolled drinking would partly rely on WM to achieve this. This emphasises the significance of WM in controlling alcohol-related behaviours. Kopera et al. (2012) argue in agreement that WM impairment may impact subsequent decision-making and this can have implications for relapse. WM dysfunction, together with certain personality features, leads to a higher risk of alcohol abuse (Kopera et al., 2012). Findings support the notion that WM requires longer periods of abstinence to show marked improvement in alcoholic-dependent patients (Velayudhan \& Saraswathy, 2020). A comparison of cognitive functions of abstaining alcohol-dependent male patients and healthy controls indicated that patients who were sober for less than a year, made more errors in both attentional shifting and WM assessments than healthy controls and patients with longer periods of abstemious (Kopera et al., 2012). The rate and extent to which cognitive functions improve after recovery thus remain with unanswered questions.

Le Berre et al. (2017) describe recovery as regaining the level of cognition before alcohol abuse. Despite evidence on the different recovery levels of selective cognitive processes, some cognitive domains remain impaired even with prolonged abstinence (Le Berre, et al., 2017). While evidence indicates that recovery is possible with prolonged abstinence, some people struggle to remain abstinent which may hinder optimal recovery from AUD. Research on repeated withdrawal from alcohol shows that it adversely impacts the recovery of cognitive functions (Le Berre et al., 2017; Loeber et al., 2010). This suggests that recurrent periods of withdrawal may be hazardous for maintaining cognitive impairment and weaken the prospect of cognitive recovery (Loeber et al., 2010). Most patients diagnosed with AUD find it challenging to remain abstinent after discharge from rehabilitation centres. The prospect of recovery is mediated by adequate cognitive skills, emotional capacity, sufficient perception, and support to allow AUD patients to understand and apply the skills needed to abstain from alcohol use and to maintain it (Le Berre et al., 2017). Abstinence is easier to maintain when the patients are in a controlled environment, but they face more challenges once control necessitates autonomous motivation.

Recovery is associated with different levels of abstinence and includes various factors. Accordingly, it is argued that interventions for the rehabilitation of alcohol dependence cannot be one dimensional. Several disciplines must be involved to enable the effective management and treatment of AUD. A multidimensional approach is thus needed. In clinical practice, however, there is an exclusive focus on managing the social and psychological factors related to alcohol use. Alcohol-related cognitive impairments are underestimated, despite the benefits it holds in treating AUD patients. Cognitive impairment may maintain the problem of addiction because several cognitive functions, needed for recovery, are weakened which promotes issues of addictive 
behaviours (Ioime et al., 2018; Stavro et al., 2013). Neglecting the cognitive functioning of AUD patients can then hamper their ability to abstain from alcohol (Cabé et al., 2016).

Research on how alcohol abuse impacts specific cognitive domains and the extent to which abstinence can contribute to the recovery of these domains is needed. Given the importance of cognitive functioning, and the limited focus on cognitive recovery in the same AUD sample, the current study assessed the impact of alcohol abstinence on specific cognitive functions in a single group of AUD patients. The aim was to determine the extent to which certain cognitive functions recover after a period of abstinence as part of a rehabilitation programme. Recovery in the current study context includes any level of significant improvement from initial assessment to post-test assessment.

\section{Method}

\subsection{Participants}

A quantitative research approach including a pre-test-post-test design was used. The patients were sampled from a local treatment facility and had a formal diagnosis of AUD. The sample included 9 patients who completed the same battery of assessments on two different occasions. The following criteria guided the selection of the sample: English speaking; 18+; formally diagnosed with AUD. Exclusion criteria included being underage ( $<18$ years old); presenting with a history of head injuries; current or past untreated neurological disorders; and any current abuse or dependence towards other substances, including psychotropic medication. Table 1 provides a summary of the demographic information of the sample.

Table 1. Demographic information

\begin{tabular}{ll}
\hline Gender & {$[\mathrm{N}]$} \\
\hline Male & 5 \\
Female & 4 \\
\hline Race & {$[\mathrm{N}]$} \\
\hline White & 8 \\
Coloured & 1 \\
\hline Educational background & {$[\mathrm{N}]$} \\
\hline Grade 12 / Senior Certificate & 7 \\
College Certificate / Diploma & 2
\end{tabular}

\subsection{Instruments}

A demographic questionnaire and the WAIS-IV ${ }^{S A}$ was administered to patients by a registered psychometrist. The WAIS-IVSA provides advanced measures of cognitive ability and is a comprehensive measure used to assess adult intelligence suited for a South African population (JVR Africa Group, 2019). Only limited sections of the assessment were used based on the instruction from the treating psychologist. It was advised that the comprehensive WAIS-IV ${ }^{\mathrm{SA}}$ would not be suitable for the AUD patients due to the duration needed to complete all the tests included in the WAIS battery. As the patients were included in a rehabilitation programme, only the following assessments from the WAIS-IVSA were administered:

- Visuospatial attention: Block design

- Abstract reasoning: Matrix reasoning

- Working memory: Arithmetic 


\subsection{Procedure}

Each participant signed an informed consent form before data collection commenced. A psychologist was present during the assessment. The WAIS-IVSA was used during both phase 1 and 2 to assess the VA, AR, and WM functioning of AUD patients. During data collection, each patient manually completed the assessments in a designated room located at the treatment facility. Only one patient was assessed at a time and the average time of completion per participant was approximately 30 minutes. The pre-assessment constituted the baseline measure before the treatment, including the abstinence component, and was administered 3-4 days after admission. The reasoning was that patients needed to detox first before assessments could commence. The post-test measurement was conducted 14 days later, a few days before discharge.

\section{Results}

Patients' cognitive assessment scores from phase 1 and phase 2 was calculated using SPSS version 27. The descriptive results are discussed to provide an overview of changes from phase 1 to phase 2. The results from the paired samples $t$-test detail the significance of the differences revealed before and after abstinence.

\subsection{Visuospatial attention: Phase 1 And Phase 2}

The maximum allowable score for the Block Design test is 66. Patients' scores ranged between 24 and $40(\mathrm{M}=32.30, \mathrm{SD}=7.26)$ for Phase 1 , baseline assessment. The standard error of the mean (SEM) was 2.3 and margin of error was $32.3 \pm 4.499( \pm 13.93 \%)$ at $95 \%$ CI. The patient group had completion times between 7 and 14 minutes against a maximum allowable completion time of 16 minutes and 30 seconds $(\mathrm{M}=8.67, \mathrm{SD}=1.50)$ at $95 \% \mathrm{CI}$ and the margin of error was $8.6667 \pm 0.98( \pm 11.31 \%)$ with a SEM of .50.

The second phase results show that the patients' scores, on average, increased $(\mathrm{M}=36.67, \mathrm{SD}=6.98)$. The SEM is 2.33 and the margin of error at $95 \% \mathrm{CI}$ is $37.6667 \pm 4.562$ $( \pm 12.11 \%)$. The average completion time was $(\mathrm{M}=8.78, \mathrm{SD}=2.64)$. The $\mathrm{SEM}$ is 0.88 and at $95 \%$ confidence interval the margin of error is $8.7778 \pm 1.722( \pm 19.61 \%)$. The significance of the difference is based on subsequent t-test analysis.

\subsection{Abstract reasoning: Phase 1 And Phase 2}

The lowest score was 7, and the highest was 15 . Patients scored on average $(M=10.89$, $S D=3.14)$. The SEM is 1.28 and the margin of error is $10.8889 \pm 2.052( \pm 18.84 \%)$ at $95 \%$ CI. The completion times ranged between 3 and 11 minutes $(M=6.00, S D=3.08)$. The standard deviation shows that the time taken to complete the tasks were widely dispersed with a range of 8 . The SEM is 1.03 with a margin of error of $6 \pm 2.014( \pm 33.56 \%)$ at $95 \% \mathrm{CI}$.

During phase 2, the highest score was 19, and the lowest was 7 with an average performance of $(M=11.67, S D=3.84)$. The $S E M$ is 1.28 giving a margin of error of $11.6667 \pm 2.509$ $( \pm 21.51 \%)$ at $95 \% \mathrm{CI}$. The average completion time was also faster compared to phase $1(\mathrm{M}=5.44$, $\mathrm{SD}=2.07)$. The $\mathrm{SEM}$ is 0.69 with a margin of error of $5.4444 \pm 1.351( \pm 24.82 \%)$ at $95 \% \mathrm{CI}$.

\subsection{Working memory: Phase 1 and Phase 2}

For WM assessment during phase 1, the lowest score against a possible total of 22 was 7, with the highest being 13, with an average performance of $(\mathrm{M}=10.44, \mathrm{SD}=2.13)$. At 95\% $\mathrm{CI}$ and 
a SEM of 0.71 the margin of error is $10.4444 \pm 1.39( \pm 13.31 \%)$. The completion time for phase 1 ranged between 3 and 12 minutes with an average completion time (M=7.44, SD=2.88). The SEM is 0.96 giving a margin of error of $7.4444 \pm 1.88( \pm 25.25 \%)$ at $95 \% \mathrm{CI}$.

During phase 2, the lowest score was 10 and the highest was 15 . Patients' average performance increased during phase $2(\mathrm{M}=11.56, \mathrm{SD}=1.59)$. The SEM is 0.53 and the margin of error at $95 \% \mathrm{CI}$ is $37.6667 \pm 4.562( \pm 12.11 \%)$. The average completion time during phase 2 was also slighter faster compared to phase $1(\mathrm{M}=6.33, \mathrm{SD}=2.35)$. The $\mathrm{SEM}$ is 0.78 and the margin of error is $6.3333 \pm 1.532( \pm 24.19 \%)$ at $95 \% \mathrm{CI}$.

\subsection{Within-subject analysis}

There was a statistically significant difference in the VA scores of patients when comparing phase 1 and $2(\mathrm{M}=7.11, \mathrm{SD}=2.07), \mathrm{t}(8)=3.42, \mathrm{p}=.009$. This means that after the period of abstinence, the patients' VA scores improved. The mean increase in the VA scores was 7.11 with a $95 \%$ CI ranging from -4.7918 to 4.7918 . The eta squared statistic $(\eta 2=1.14)$ indicated a large effect size (Pallant, 2016). Cohen's guideline was used to interpret the observed effect sizes. The general guidelines for Cohen's d are small $=0.20$, medium $=0.50$, and large $=0.80$ (Cohen, 1992; Pallant, 2016). This indicates that the magnitude of the difference is large. It is acknowledged that the interval is wide and contains $o$ indicating a poor estimate of the population value. This means that it is possible that no significant difference will be found should the same study be repeated. The significant difference within the current study parameters should thus be interpreted with caution.

There was no significant difference in patients' AR scores when comparing phase 1 and $2(\mathrm{M}=0.77, \mathrm{SD}=1.29), \mathrm{t}(8)=.59, \mathrm{p}=.566$. This means that after the period of abstinence, the patients' scores did not significantly improve. The eta squared statistic $\left(\eta^{2}=0.2\right)$ indicated a small effect size (Cohen, 1992).

No significant difference in the WM scores of patients were observed when comparing phase 1 and $2(\mathrm{M}=1.11, \mathrm{SD}=.67), \mathrm{t}(8)=1.64, \mathrm{p}=.138$. After a short period of abstinence, the patients' WM abilities did not seem to improve. The eta squared statistic $\left(\eta^{2}=0.55\right)$ indicated a medium effect size (Cohen, 1992).

\section{Discussion}

Research has demonstrated varying degrees of impairment in prominent cognitive functions due to alcohol abuse. The extent to which this impairment improves after a period of abstinence is not yet clear. Alcoholism is described as a multifaceted dynamic disorder (Le Berre et al., 2017) that includes periods of abstinence and relapse. Extensive research has demonstrated that alcohol abuse diminishes important cognitive functions (Ahmadi et al., 2013; Bruijnen et al., 2021; Devere, 2016; Fein et al., 2006; Le Berre et al., 2017; Stavro et al., 2013; Zehra et al., 2019). Velayudhan and Saraswathy (2020) emphasise the value of understanding how cognitive functions, amongst alcoholic dependent patients, change within different phases of treatment. This may enhance the understanding of the functions that improve with shorter periods of abstinence compared to those functions that continue to demonstrate deficits. Findings suggest that VA abilities are particularly vulnerable to alcohol abuse (Fein et al., 2006; Ioime et al., 2018; Mocaiber et al., 2011; Stavro et al., 2013). Similarly, AR abilities also show deterioration in relation to alcohol use (Velayudhan \& Saraswathy, 2020). Cognitive functioning may recover after a prolonged period of abstinence, ranging from six months to one year, given that there was no relapse (Ioime et al., 2018; Le Berre et al., 2017; Velayudhan \& Saraswhathy, 2020). The 
improvement of cognitive performance after alcohol abuse demonstrates the plasticity of the brain in that neural structures are capable of repair.

Some cognitive functions may show improvement within a few weeks, while others may require longer periods of recovery. WM ability, for example, show long-term deficits. Velayudhan and Saraswathy (2020) maintain that the harm caused by alcohol abuse may be irreversible in relation to WM. Desphande (2015) and Fein et al. (2006) agree that WM abilities are significantly compromised by alcohol abuse.

Ioime et al. (2018) explained that few studies examine the same sample of alcohol impaired participants to explore changes over time. The current study observed the same group of AUD patients on two different occasions. The baseline assessment was conducted a few days after admission giving patients time to detox as they often arrive intoxicated at the rehabilitation facility. The post-test measure was completed 14 days later, a few days before discharge. The findings revealed significant improvement in the VA abilities of the patients when phase 1 and phase 2 was compared. Patients also completed the VA tasks faster during phase 2. The small sample size in conjunction with the short period of abstinence limits the extent to which conclusive arguments can be made. The findings contradict previous research on VA, where Zehra et al. (2019) for example, found no behavioural differences with regard to VA when compared with healthy controls. Their findings did, however, support lower activation of brain regions associated with attention as evidenced by fMRI scans. The findings from Fein et al. (2006) are comparable to Zehra et al. (2019) and show that despite long-term periods of sobriety, deficits in spatial processing persist. Similar to the current study, the results from several other studies showed improvement in the attentional processes and executive functions of the patients (Cabé, et al., 2016; Fein, et al., 1990; Fein \& Cardenas, 2015; Kopera et al., 2012; Pelletier, et al., 2016). Ioime et al. (2018) explored the longitudinal effect of abstinence and the findings showed that most cognitive functions improved with longer periods of sobriety (i.e., six months to one year), however, VA abilities did not improve and may only recover after more than one year of abstinence.

The impact of alcohol abuse is also apparent in the WM and AR ability with AUD patients presenting with marked difficulty in memory and problem-solving abilities. In this study, no significant differences were found in the WM abilities or AR abilities of patients when phase 1 and phase 2 data was compared. Kopera et al. (2012) echoed these results maintaining that alcohol dependent participants presented with lower WM capacity in comparison to healthy controls. WM is an essential part of decision-making and deficits may impair AUD patients' ability to effectively manage and control their drinking, increasing the prospect of relapse (Kopera et al., 2012; Le Berre et al., 2017). Diminished cognitive functioning, concerning AR, also suggest that problem drinking may persist due to inadequate skills regarding problem-solving. Ioime et al. (2018) argued that impaired executive functions mean that alcohol dependent participants are unable to learn by negative experiences thereby maintaining their addiction. The diverse nature of AUD and the impact on cognition means that some cognitive functions may improve with short periods of abstinence, while others like WM, need substantial time to improve (Velayudhan \& Saraswathy, 2020). Deshpande (2015) explains that WM may be permanently impaired in some cases of alcohol abuse. The length of alcohol abuse and abstinence ultimately predicts the extent of impairment and the potential recovery of cognitive deficits. The short period of abstinence in the current study may have limited the extent to which recovery of WM and AR abilities could be observed. Similar to previous findings, these cognitive domains may require longer periods of abstinence to show marked improvements. 


\subsection{Practical implications}

The findings hold both theoretical and practical value. Theoretically, the findings contribute to improving the understanding of several cognitive functions and the degree of impairment and recovery, following a short period of abstinence. Practically, the findings emphasise the need for interventions to consider the importance of cognition, both in assessment and treatment of AUD patients. The significance of cognitive functioning is grounded in the mediating role it plays in preventing relapse (Velayudhan \& Saraswathy, 2020). Ahmadi et al. (2013) also emphasise the significance of AUD research that enhances our understanding of the psychological and neurological aspects valuable to both public health and addiction disorders. Le Berre et al. (2017) echoed this by describing the multidimensional nature of alcoholism and its effects. The ability to recover from AUD and relapse prevention, is dependent on sound cognitive functioning. Patients need to be able to cope with stressful demands and be able to achieve longterm sobriety. The ability to stop drinking, and to maintain this change requires multiple neurological functions, social cognition, emotional support and adequate cognitive control (Le Berre et al. 2017). Enhanced understanding of cognition is necessary to improve cognitive behavioural therapy (CBT) approaches, thereby aiding behavioural change.

There has been debates about the level of cognitive improvement amongst alcoholdependent patients. This study makes a valuable contribution by adding to the body of knowledge on how cognitive functions change within the same sample of AUD patients based on a short period of abstinence. The findings also provide support for the value of assessing cognitive functions and including a treatment focused on recovering cognitive functions as part rehabilitation programmes. This will not only improve patients' cognitive abilities but can also help patients prevent or minimise relapse and empower them to maintain sobriety.

\section{Conclusion}

In conclusion, cognitive impairment compromises the success of psychosocial treatment and identifying cognitive deficits is essential with regard to rehabilitation (Ioime et al., 2018; Kopera et al. 2012). The importance of cognitive assessment in AUD patients is thus valuable for both clinical and practical application. Insufficient levels of cognitive functioning can impede AUD patients' prospects of recovery and increase the likelihood of relapse.

Cognitive assessment and rehabilitation approaches should thus be included in the treatment programmes of AUD patients. No significant differences were observed in the WM and AR abilities of the current AUD patient sample. Significant improvement was found in the VA functioning of the patients, although the small sample size and study limitations cautions against any definitive conclusions. The findings provide support for the value of abstinence in cognitive recovery and the significance of incorporating cognitive rehabilitation in intervention strategies (Devere, 2016). A multi-disciplinary approach that deals with psycho-affective, behavioural, and cognitive consequences, is thus needed when treating AUD.

\subsection{Limitations and future directions}

The present study included a restricted number of AUD patients. The small sample size limits the extent to which conclusive arguments can be made. The period of abstinence was short, constituting 14 days of abstinence. Factors that are known to impact alcohol use like, age; period of alcohol abuse; and frequency of relapse, were not considered in the current investigation. COVID-19 regulations prevented the inclusion of a third assessment. Future studies should include a larger sample of AUD patients and assess cognitive impairment and improvement across several periods of recovery. It is suggested that longer periods of abstinence are needed to show 
E. E. Mofokeng \& S. N. Mostert - Evaluating the Impact of Alcohol Abstinence...

improvement in prominent cognitive functions. It is also recommended that factors related to period of alcohol use, frequency and quantity also be taken into consideration.

\subsection{Ethical consideration}

The Faculty of Humanities Research Ethics Committee at the University of Pretoria granted ethical approval for the study. All participants received an information sheet including the purpose of the study. The research procedure was adequately communicated and data collection was done by a registered psychometrist. Informed consent was obtained by all participants before data collection commenced. Voluntary participation was emphasised and participants had the right to withdraw from the study without any negative consequences. Confidentiality was ensured as no personal information was connected to the data. The procedures performed in this study were based on the ethical standards provided by the Faculty of Humanities Research Ethics Committee.

\section{Acknowledgements}

This research did not receive any specific grant from funding agencies in the public commercial, or not-for-profit sectors.

The authors declare no competing interests.

\section{References}

Ahmadi, A., Pearlson, G. D., Meda, S.A., Dager, A., Potenza, M. N., Rose, R., Austad, C. S., Raskin, S. S., Fallahi, C. R., Tennen, H., Wood, M. R., \& Stevens, M. C. (2013). Influence of alcohol use on neural response to Go/No-Go task in college drinkers. Neuropsychopharmacology, 38, 21972208. https://doi.org/10.1038/npp.2013.119

Astorga, M. L. (2013). Alcoholism and conditional reasoning: difficulties in specific mental domains or in the general use of heuristics? Psychologica Belgica, 53(4), 3-16. https://doi.org/10.5334/pb5.3-4-3

Bagga, D., Singh, N., Singh, S., Modi, S., Kumar, P., Bhattacharya, D., \& Garg, M. (2014). Assessment of abstract reasoning abilities in alcohol-dependent subjects: an fMRI study. Neuroradiology, 69-77. https://doi.org/10.1007/s00234-013-1281-3

Banerjee, N. (2014). Neurotransmitters in alcoholism: A review of neurobiological and genetic studies. Indian Journal of Human Genetics, 2O(1), 20-31.

Bates, M. E., Buckman, J. F. \& Nguyen, T. T. (2013). A role for cognitive rehabilitation in increasing the effectiveness of treatment for alcohol use disorders. Neuropsychol Rev, 23(1), 27-47. https://doi.org/10.1007/s11065-013-9228-3

Batman, A. M. (2015). Translating alcohol research: opportunities and challenges. Alcohol Research, 37(1), 7-14. https://www.ncbi.nlm.nih.gov/pmc/articles/PMC4476605/

Bernadin, F., Maheut-Bosser, A. \& Paille, F. (2014). Cognitive impairments in alcohol-dependent subjects. Frontiers in Psychiatry, 5(78), 1-6. https://doi.org/10.3389/fpsyt.2014.00078

Brennan, S. E., McDonald, S., Page, M. L., Reid, J., Ward, S., Forbes, A. B., \& McKenzie, J. E. (2020). Longterm effects of alcohol consumption on cognitive function: a systematic review and doseresponse analysis of evidence published between 2007 and 2018. Systematic Reviews, 9(33), 1-39. https://doi.org/10.1186/s13643-019-1220-4 
Breukelaar, I. A., Antees, C., Grieve, S. M., Foster, S. L., Gomes, L., Williams, L. M., et al. (2017). Cognitive control network anatomy correlates with neurocognitive behavior: A longitudinal study. Hum. Brain Mapp. 38, 631-643. https://doi.org/10.1002/hbm.23401

Bruijnen, C.J.W.H.S., Walvoort, J.W., Dijkstra, B.A.G., de Jong, C.A.J., \& Kessels, R.P.C. (2021). The course of cognitive performance during inpatient treatment in patients with alcohol use disorder with no, mild or major neurocognitive disorders. Alcohol and Alcoholism, 56(1), 89-100. https://doi.org/10.1093/alcalc/agaa100

Buddy, T. (2020). Alcohol effects that last long into sobriety. https://www.verywellmind.com/alcoholeffects-last-long-into-sobriety-66610

Cabé, N., Laniepce, A.., Ritz, L., Lannuzel, C., Boudehent, C., Vabret, F., Eustache, F., Beaunieux, H., \& Pitel, A. L. (2016). Cognitive impairments in alcohol dependence: From screening to treatment improvements. Encephale, 42(1), 74-81. https://doi.org/10.1016/j.encep.2015.12.012

Chai, W. J., Hamid, A. I. A. \& Abdullah, J. M. (2018). Working memory from the psychological and neurosciences perspectives: A Review. Frontiers in Psychology, 9(401), 1-16. https://doi.org/10.3389/fpsyg.2018.00401

Cohen, J., (1992). Statistical Power Analysis. Current Directions in Psychological Science, 1(3), 98-101.

Cowan, N. (2014, June). Working Memory Underpins Cognitive Development, Learning, and Education. Educ Psychol Rev., 26(2), 197-223. https://doi.org/10.1007/s10648-013-9246-y

do Canto-Pereira, L. H. M., David, I. de P., Machado-Pinheiro, W., \& Ranvaud, R. D. (2007). Effects of acute alcohol intoxication on visuospatial attention. Human \& Experimental Toxicology, 26(4), 311319. https://doi.org/10.1177/0960327106070490

Deshpande, S.S. (2015). Differential effects of alcohol consumption behaviours on working memory processes. Journal of European Psychology Students, 6(3), 14-23. http://dx.doi.org/10.5334/jeps.dd

Devere, R. (2016, October). The cognitive consequences of alcohol use. https://practicalneurology.com/articles/2016-oct/the-cognitive-consequences-of-alcohol-use

Doruk, D., Chanes, L., Malavera, A., Merabet, L. B., Valero-Cabré, A., \& Fregni, F. (2018). Cross-modal cueing effects of visuospatial attention on conscious somatosensory perception. Heliyon, 4(4), e00595. https://doi.org/10.1016/j.heliyon.2018.e00595

Erdozain, A.M., Morentin, B., Bedford, L., King, E., Tooth, D. et al. (2014). Alcohol-Related Brain Damage in Humans. PLOS ONE, 9(4), e93586. https://doi.org/10.1371/journal.pone.0093586

Fein, G., Torres, J., Price, L.J., \& Di ScIafani, V. (2006). Cognitive performance in long-term abstinent alcoholics. Alcohol Clin Exp Res, 30(9), 1538-1544

Fein, G., Bachman, L., Fisher, S. \& Davenport, L., (1990). Cognitive impairments in abstinent alcoholics. Western Journal of Medicine, 152(5), 531-537.

Fein, G., \& Cardenas, V. A. (2015). Neuroplasticity in human alcoholism: studies of extended abstinence with potential treatment implications. Alcohol Research, 37(1), 125-141.

Ioime, L., Guglielmo, R., Affini, G.F., Quatrale, M., Martinotti, G., Callea, A., Savi, E., \& Janiri, L. (2018). Neuropsychological performance in alcohol dependent patients: A one-year longitudinal study. Psychiatry Investigation, 15(5), 505-513. https://doi.org/10.30773/pi.2017.09.27.1

JVR Africa Group. (2019). Wechsler Adult Intelligence Scale® - Fourth SA Edition (WAIS®-IV SA). https://jvrafricagroup.co.za/catalogue/assessment/wechsler-adult-intelligence-scale-fourthsa-edition-wais-iv-sa/.

Kopera, M., Wojnar, M., Brower, K., Glass, J., Nowosad, I., Gmaj, B., \& Szelenberger, W. (2012). Cognitive functions in abstinent alcohol-dependent patients. Alcohol, 46(7), 665-71. https://doi.org/10.1016/i.alcohol.2012.04.005

Le Berre, A.P., Fama, R., \& Sullivan, E. V. (2017). Executive Functions, memory, and social cognitive deficits and recovery in chronic alcoholism: A critical review to inform future research. Alcoholism, Clinical and Experimental Research, 41(8), 1432-1443. https://doi.org/10.1111/acer.13431 
E. E. Mofokeng \& S. N. Mostert - Evaluating the Impact of Alcohol Abstinence...

Lechner, W. V., Day, A. M., Metrik, J., Leventhal, A. M., \& Kahler, C. W. (2016). Effects of alcohol-induced working memory decline on alcohol consumption and adverse consequences of use. Psychopharmacology, 233(1), 83-88. https://doi.org/10.1007/s00213-015-4090-z

Loeber, S., Duka, T., Welzel Márquez, H., Nakovics, H., Heinz, A., Mann, K., \& Flor, H. (2010). Effects of repeated withdrawal from alcohol on recovery of cognitive impairment under abstinence and rate of relapse. Alcohol Alcohol, 45(6), 541-547. https://doi.org/10.1093/alcalc/agq065

Mocaiber, I., David, I.A., de Oliveira, L., Pereira, M.G., Volchan, E., Figueira, I., Vila, J., \& MachadoPinheiro, W. (2011). Alcohol, emotion and attention: Revisiting the Alcohol Myopia Theory, 24, 403-410. https://doi.org/10.1590/So102-79722011000200022

Molnar, S.M., Beaton, L.E., Happer, J.P., Holcomb, L.A., Huang, S., Arienzo, D., \& Marinkovic, K. (2018). Behavioral and brain activity indices of cognitive control deficits in binge drinkers. Brain Sci, 8(1), 1-19. https://doi.org/10.3390/brainsci8010009

Oscar-Berman, M., Valmas, M. M., Sawyer, K. S., Ruiz, S. M., Luhar, R. B., \& Gravitz, Z. R. (2014). Profiles of impaired, spared, and recovered neuropsychologic processes in alcoholism. Handbook of clinical neurology, 125, 183-210. https://doi.org/10.1016/B978-0-444-62619-6.00012-4

Pallant, J. (2016). SPSS Survival Manual: A step by step guide to data analysis using SPSS Program. (6th ed.). London, UK: McGraw-Hill Education.

Pelletier, S., Nalpas, B., Alarcon, R., Rigole, H., \& Perney, P. (2016). Investigation of cognitive improvement in alcohol-dependent inpatients using the montreal cognitive assessment (MoCA) Score. Journal of addiction, 2016(1539096), 1-7. https://doi.org/10.1155/2016/1539096

Saults, J., Cowan, N., Sher, K., \& Moreno, M. (2007). Differential effects of alcohol on working memory: distinguishing multiple processes. Exp Clin Psychopharmacol, 15(6), 576-587. https://doi.org/10.1037/1064-1297.15.6.576

Stavro, K., Pelletier, J., \& Potvin, S. (2013). Widespread and sustained cognitive deficits in alcoholism: a meta-analysis. Addiction Biology, 18, 203-213. https://doi.org/10.1111/j.13691600.2011.00418.x

Velayudhan, R., \& Saraswathy, S. M. (2020). Neuropsychological functions in short- and long-term alcohol abstinence. Ind $J$ Priv Psychiatry; 14(1), 26-29. https://doi.org/10.5005/jp-journals-100670054

Woods, A. J., Porges, E. C., Bryant, V. E., Seider, T., Gongvatana, A., Kahler, C. W., de la Monte, S., Monti, P. M., \& Cohen, R. A. (2016). Current heavy alcohol consumption is associated with greater cognitive impairment in older adults. Alcohol Clin Exp Res, 4O(11), 2435-2444. https://doi.org/10.1111/acer.13211.

World Health Organization (WHO). (2021). Alcohol. https://www.who.int/healthtopics/alcohol\#tab=tab 1.

Zehra, A., Lindgren, E., Wiers, C. E., Freeman, C., Miller, G., Ramirez, V., Shokri-Kojori, E., Wang, G., Talagala, L., Tomasi, D., \& Volkow, N. D. (2019). Neural correlates of visual attention in alcohol use disorder. Drug and Alcohol Dependence, 194, 430-437. https://doi.org/10.1016/j.drugalcdep.2018.10.032.

Zirnsak, M., \& Moore, T. (2015). The what and where of visual attention. Neuron, 88(4), 626-8. https://doi.org/10.1016/j.neuron.2015.11.005 